\section{Les protéines SIBLING}

\section{Outils moléculaires de la progression tumorale et de l'angiogenèse}

$>$ La famille SIBLING comprend cinq membres: l'ostéopontine (OPN), la sialoprotéine osseuse (BSP), la protéine matricielle de dentine l (DMPl), la sialophosphoprotéine de dentine (DSPP) et la phosphoglycoprotéine de matrice extracellulaire (MEPE). Ces protéines ont été regroupées sur la base de leurs caractéristiques biochimiques et génétiques communes. D’abord considérées comme spécifiques des tissus minéralisés de l'os et de la dent, leur surexpression a été ensuite mise en évidence au niveau d'une large variété de tumeurs chez l'homme. Dans cette revue, nous décrivons les rôles attribués aux protéines SIBLING au niveau de chacune des étapes de la progression cancéreuse et métastatique, ainsi que de l'angiogenèse. <

\section{Les protéines de la famille SIBLING}

Le regroupement de cinq protéines des matrices minéralisées de l'os et de la dent au sein d'une famille appelée SIBLING (small integrin-binding ligand N-linkedglycoprotein) a été proposé pour la première fois par L.W. Fisher et al. [1] sur la base de leurs caractéristiques biochimiques et génétiques communes. Les principaux membres de cette famille sont l'ostéopontine (OPN), la sialoprotéine osseuse (BSP), la sialophosphoprotéine de dentine (DSPP), la protéine matricielle de dentine 1 (DMP1) et la phosphoglycoprotéine de matrice extracellulaire (MEPE). Ces protéines, hautement glycosylées et phosphorylées, ont d'abord été isolées à partir des matrices extracellulaires minéralisées des os et de la dent. Alors que l'OPN est la protéine SIBLING la mieux étudiée, les autres protéines ont connu un regain d'intérêt ces 20 dernières années, surtout après la mise en évidence de leur expression ectopique en dehors des tissus minéralisés et, notamment, au niveau des cellules tumorales malignes. L'objet de cette revue est de décrire les propriétés des protéines SIBLING dans le

Vignette (Photo (c) Inserm - Nicolas Ricard et Didier Grunwald).

\section{Virginie Lamour, Marie-Julie Nokin, Aurélie Henry, Vincent Castronovo, Akeila Bellahcène}

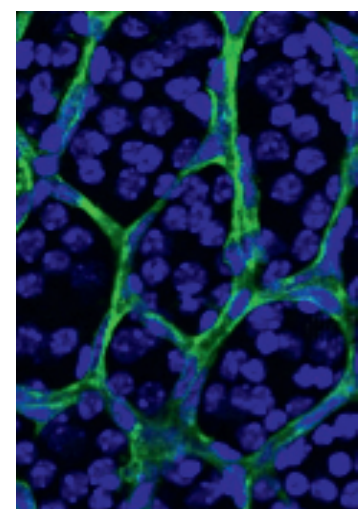

Laboratoire de recherche sur les métastases, GIGA (groupe interdisciplinaire de génoprotéomique appliquée) -Cancer, Université de Liège, Building 23, Sart Tilman, 4000 Liège, Belgique. a.bellahcene@ulg.ac.be

contexte du cancer. Ainsi, leur expression importante par différents types de cancers chez l'homme (pour une revue détaillée, voir [2]) ne sera mentionnée ici que dans quelques exemples utiles.

Chez l'homme, les gènes codant pour les SIBLING sont localisés au niveau d'une région de 375000 paires de bases sur le bras long du chromosome 4. L.W. Fisher et al. ont observé que ces gènes présentent de nombreuses similitudes, notamment au niveau de l'agencement de leurs exons (Figure 1), suggérant qu'ils proviendraient d'un même gène ancestral ayant subi des duplications et des modifications au cours du temps [1].

Les cinq protéines partagent plusieurs domaines fonctionnels, tels que des sites de liaison au calcium, le motif RGD (Arg-Glyc-Asp) qui assure l'interaction entre ces protéines et les récepteurs de surface cellulaire de type intégrine, et le domaine appelé ASARM (acidic serine and aspartate-rich motif) qui est impliqué dans la minéralisation osseuse (Figure 1). D'autres domaines sont plus spécifiques de certaines SIBLING comme la séquence cryptique SVVYGLR (Figure 1) qui permet la liaison de l'OPN aux intégrines $\alpha_{9} \beta_{1}$ et $\alpha_{4} \beta_{1}$. De manière générale, ces protéines lient les récepteurs de type intégrine et le récepteur CD44 ${ }^{1}$ (Tableau I). Ces interactions activent des voies de signalisation aboutissant à l'adhésion, la migration et la survie cellulaires.

Les activités biologiques des SIBLING sont modulées par des processus protéolytiques qui peuvent révéler des sites de liaison cryptiques et/ou supprimer des domaines fonctionnels influençant, notamment, l'adhésion et la migration cellulaires. Les fonctions connues des SIBLING au

${ }^{1}$ CD44 désigne une famille de protéines résultant de l'épissage alternatif de 10 exons du gène, fonctionnant dans la réponse immune et comme récepteur de l'acide hyaluronique [51]. 

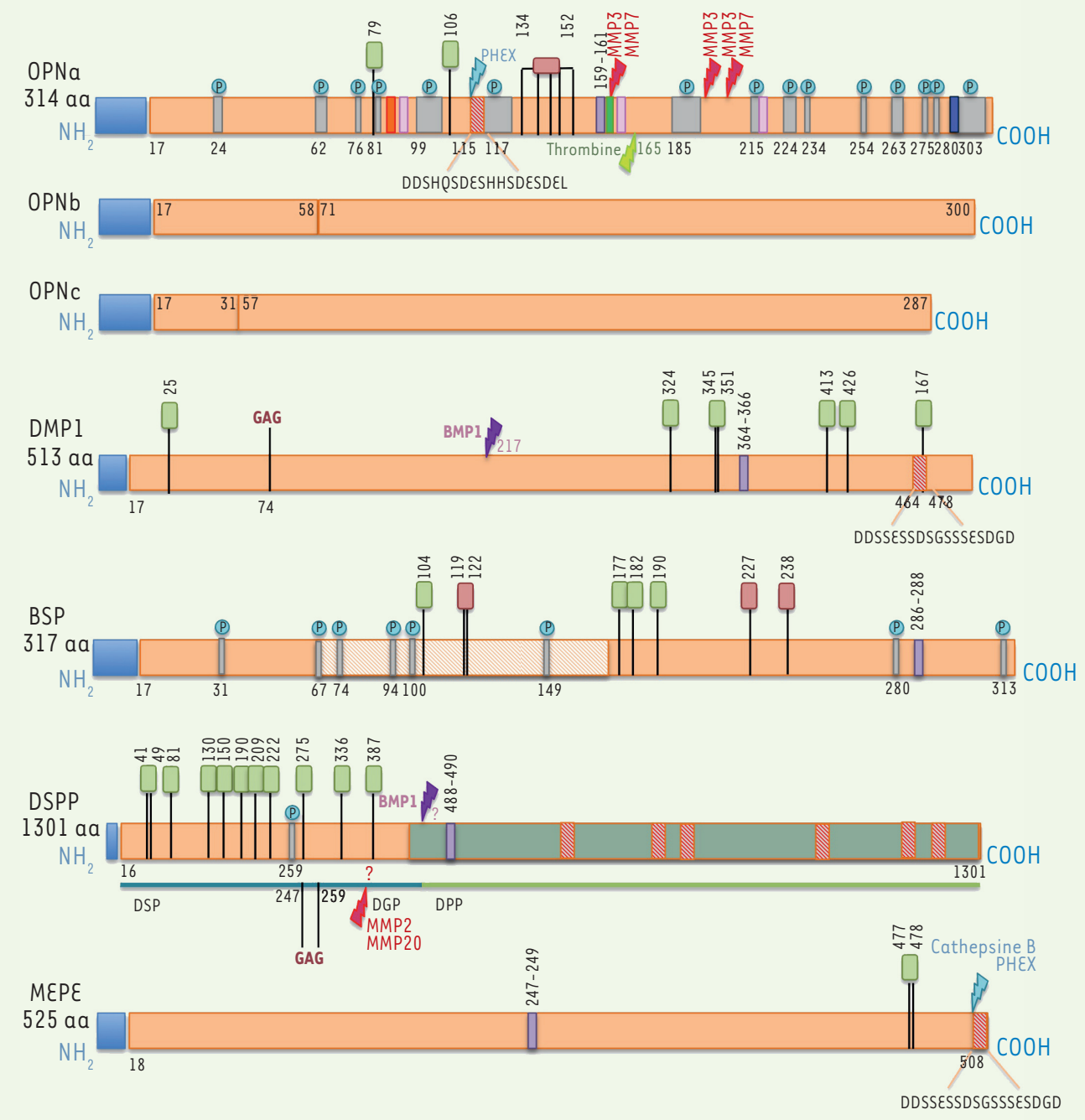

\begin{tabular}{|lll|}
\hline Motif ASARM & Domaine de liaison au calcium & $\begin{array}{l}\text { Domaine de liaison aux } \\
\text { cristaux d'hydroxyapatite }\end{array}$ \\
\hline Région riche en Asp-Ser & Motif RGD & N-glycosylation \\
\hline Région riche en Asp-Glu & Motif SVVYGLR & D-glycosylation \\
PAG Site pour glycosaminoglycane & Domaine de liaison à l'héparine enzymatique & \\
\hline
\end{tabular}

Figure 1. Principaux domaines structuraux et modifications post-traductionnelles potentielles des SIBLING. Cette famille comprend l'ostéopontine (OPN), la protéine matricielle de dentine (DMP1), la sialoprotéine osseuse (BSP), la sialophosphoprotéine de dentine (DSPP) et la phosphoglycoprotéine de dentine (MEPE). L'OPN possède deux isoformes obtenues par épissage alternatif: les OPNb et c. Les exons 5 et 4 sont absents dans I'OPNb et I'OPNc, respectivement. La séquence en acides aminés des protéines SIBLING est particulièrement riche en résidus de nature acide (régions riches en Asp-Ser et Asp-Glu). Ces protéines présentent plusieurs domaines communs, tels que les motifs RGD (Arg-Gly-Asp) et la séquence cryptique SVVYGLR de liaison aux intégrines. Le motif ASARM (acidic serine aspartate-rich MEPE-associated motif), les domaines de liaison à l'hydroxypatite et les sites de clivage par PHEX (phosphate-regulating endopeptidase homologue, X-linked) impliqués dans le processus de minéralisation sont indiqués. L'activité des SIBLING est régulée par des modifications post-traductionnelles, telles que la phosphorylation, la glycosylation et/ou l'addition d'un glycosaminoglycane (GAG). Enfin, le clivage des SIBLING par des enzymes, telles que la thrombine et la BMPI (protéine morphogénétique osseuse 1), génère des fragments protéiques dotés de propriétés spécifiques. Aa : acide aminé. 


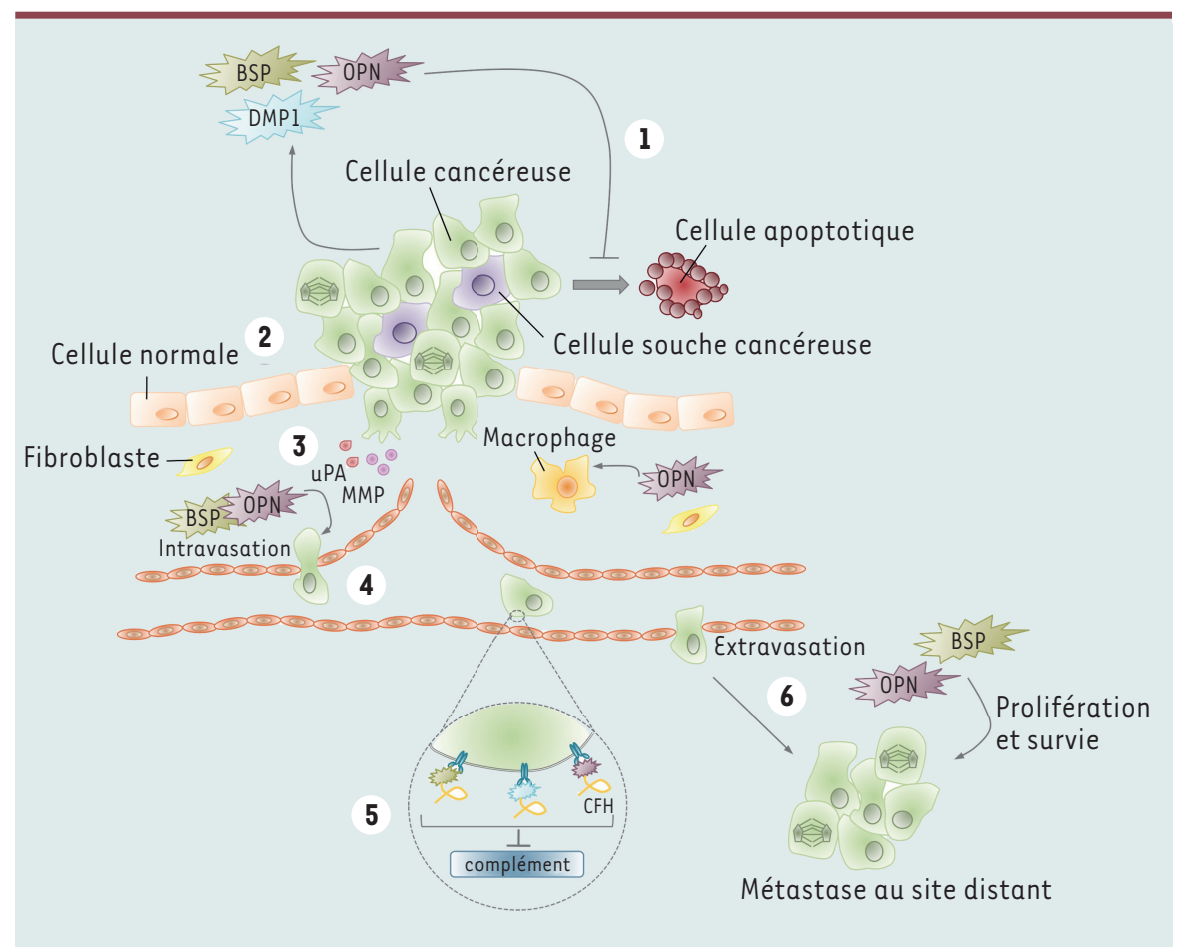

Figure 2. Rôles des protéines SIBLING dans les différentes étapes de la progression cancéreuse et métastatique. Étapes 1-2: au site primaire, les protéines SIBLING sécrétées par les cellules cancéreuses et par les cellules du stroma se lient aux récepteurs de surface cellulaire intégrines et CD44. Cette liaison conduit à l'activation de voies de signalisation qui contrôlent la survie des cellules cancéreuses et leur prolifération. Étape 3 : les cellules cancéreuses, qui se détachent de la tumeur primaire, dégradent la membrane basale et envahissent le stroma avoisinant. Les MMP (matrix metalloproteinase) et l'uPA (urokinase plasminogen activator) assurent la protéolyse de la matrice extracellulaire. L'OPN induit l'activation de l'uPA et des MMP, la motilité cellulaire et l'invasion. De plus, les SIBLING peuvent activer des MMP spécifiques (BSP-MMP2, OPN-MMP3 et DMP1-MMP9). L'OPN agit également comme une molécule chimio-attractive pour les macrophages et promeut leur infiltration au sein de la tumeur. Étape $4:$ afin de former des tumeurs secondaires à des sites distants, les cellules tumorales empruntent, notamment, la circulation sanguine. Étape 5 : le transport des cellules cancéreuses dans la circulation est une étape critique pour la formation de métastases car celles-ci sont confrontées au système immunitaire de l'hôte. La BSP, la DMPl et l'OPN sécrétées par les cellules cancéreuses protègent ces dernières de la lyse par le système du complément (Facteur $\mathrm{H}$ du complément, $\mathrm{CFH}$ ). Étape 6 : au site distant, les cellules cancéreuses s'extravasent pour former une colonie secondaire. L'expression des SIBLING par la colonie métastatique nouvellement formée va favoriser sa prolifération et sa survie au travers de mécanismes similaires à ceux observés au niveau du site primaire.

niveau des matrices extracellulaires minéralisées sont détaillées dans une revue récente [3]. L'existence d'isoformes contribue également à la diversité des fonctions attribuées aux SIBLING. Par exemple, trois isoformes de l'OPN ( $a, b$ et $c$ ) ont été décrites et étudiées pour leurs fonctions au niveau des cellules cancéreuses (Figure 1).

La régulation transcriptionnelle des gènes SIBLING a été particulièrement bien étudiée dans le contexte des différenciations ostéoblastique et odontoblastique. Les promoteurs des gènes codant pour I'OPN, la BSP, Ia DMPI et la DSPP contiennent de nombreuses séquences consensus, telles que les sites de liaison aux facteurs de transcription API (activator protein 1) et NFKB. Runx2 (runt-related transcription factor 2) est un facteur de transcription clé pour la régulation des gènes impliqués dans la biologie de l'os et, de ce fait, il est aussi un élément régulateur crucial de l'expression des SIBLING. Toutes les protéines Runx sont intimement associées à la progression tumorale, l'invasion et la formation de métastases. De manière intéressante, Runx2 active l'expression de I'OPN et de la BSP dans les cellules cancéreuses mammaires humaines, suggérant que l'expression des SIBLING est soumise à la même régulation transcriptionnelle au niveau des cellules osseuses et des cellules cancéreuses (pour revue, voir [2]). D'autres familles de facteurs de transcription associés à la progression tumorale régulent l'expression de I'OPN dans des modèles de cancer du sein, de mélanome et de leucémie (pour revue, voir [4]). Des études détaillées du promoteur proximal des gènes codant pour la DMPI et la DSPP sont nécessaires afin d'identifier les éléments régulateurs responsables de leur surexpression au niveau des cellules cancéreuses.

\section{Rôles des protéines SIBLING au cours de la progression tumorale et métastatique}

Dans leur article séminal, D. Hanahan et R.A. Weinberg [5] ont résumé les propriétés principales communes aux cellules cancéreuses et qui les différencient des cellules normales. Chacune de ces caractéristiques est directement ou indirectement liée au gain de capacités nouvelles permettant aux cellules cancéreuses d'acquérir un phénotype malin, prolifératif et invasif. II est tentant, à ce stade, de comparer les SIBLING à des outils qui aideraient les cellules tumorales malignes à acquérir ces caractéristiques. Ainsi, l'expression des SIBLING est associée à une prolifération cellulaire accrue, un blocage des signaux restrictifs de croissance, un échappement à l'immunité de l'hôte, et la capacité d'invasion des tissus hôtes avoisinants (Figure 2). 


\begin{tabular}{|c|c|c|c|}
\hline \multicolumn{2}{|c|}{ Récepteurs } & SIBLING & $\begin{array}{l}\text { Domaines } \\
\text { impliqués }\end{array}$ \\
\hline \multirow{8}{*}{ Intégrines } & $\alpha v \beta 1$ & OPN & RGD \\
\hline & $\alpha v \beta 3$ & OPN, BSP, DMPI & RGD \\
\hline & $\alpha v \beta 5$ & OPN, BSP, DMPI & RGD \\
\hline & $\alpha v \beta 6$ & OPN, DSPP & RGD \\
\hline & $\alpha 5 \beta 1$ & OPN & $R G D$ \\
\hline & $\alpha 8 \beta 1$ & OPN & $R G D$ \\
\hline & $\alpha 4 \beta 1$ & OPN & SVVYGLR \\
\hline & $\alpha 9 \beta 1$ & OPN & SVVYGLR \\
\hline \multirow{4}{*}{ CD44 } & CD44s & OPN, DMPI & $?$ \\
\hline & $\mathrm{CD} 44 \mathrm{v} 3$ & OPN & $?$ \\
\hline & $\mathrm{CD} 44 \mathrm{v} 6$ & OPN & $?$ \\
\hline & $\mathrm{CD} 44 \mathrm{v} 7$ & OPN & $?$ \\
\hline
\end{tabular}

Tableau I. Récepteurs de surface cellulaire liant les protéines SIBLING.

Leurs fonctions durant ces différents processus mettent en jeu une série de cascades de signalisation suite à leur liaison via des récepteurs spécifiques (Tableau I) présents à la surface des cellules cancéreuses (Figure 3).

\section{Adhésion cellulaire et prolifération}

Les cellules cancéreuses interagissent avec les protéines de la famille des SIBLING et/ou leurs multiples fragments protéolytiques grâce à différents récepteurs de type intégrine, de manière dépendante ou indépendante du motif RGD (Tableau l). Ainsi, la liaison de la BSP aux intégrines $\alpha v \beta 3$ et $\alpha v \beta 5$ stimule l'adhésion, la prolifération et la migration des cellules cancéreuses mammaires [6]. De plus, I'OPN et la DMPI interagissent avec le récepteur CD44 et certains de ses variants exprimés à la surface des cellules cancéreuses. L'OPN se lie au CD44v6 et induit la propagation de signaux cytoplasmiques activant des intégrines et la migration des cellules cancéreuses de côlon [7]. Les cellules cancéreuses mammaires exprimant le récepteur $\alpha v \beta 3$ adhèrent à la matrice osseuse enrichie en BSP, ainsi qu'à la BSP recombinante lors d'expériences d'adhésion et d'invasion [8]. Le domaine RGD de la DMPI régule l'adhésion de cellules de la pulpe dentaire et d'ostéoblastes in vitro (Figure 2, étape 1-2) [9].

II est bien établi que les protéines SIBLING favorisent la prolifération des cellules cancéreuses. L'OPN [50] stimule la prolifération des cellules cancéreuses prostatiques humaines dans un modèle de xénogreffe chez la souris [10]. Des études plus récentes ont montré que les isoformes $a$ et $b$ de l'OPN favorisent la survie et la prolifération des cellules cancéreuses [11]. Les voies de signalisation intracellulaire par lesquelles l'OPN exerce ses effets sur la prolifération et la migration ont été bien caractérisées (pour revue, voir [12]). La surexpression de la BSP au niveau des cellules cancéreuses pancréatiques stimule leur prolifération [13]. Aussi, la BSP recombinante favorise la prolifération des cellules cancéreuses mammaires in vitro [6]. Récemment, il a été démontré que la DSPP jouait également un rôle positif dans la prolifération des cellules de cancers oraux [14].

Dégradation de la matrice extracellulaire et invasion Les cellules tumorales ayant un haut potentiel migratoire et exprimant des protéases capables de dégrader la matrice extracellulaire sont susceptibles d'envahir les tissus avoisinants. Les protéines de la famille des SIBLING, surexprimées au niveau des cellules cancéreuses, jouent un rôle dans le potentiel invasif de ces cellules (Figure 2, étape 3). La surexpression de I'OPN au niveau des cellules cancéreuses prostatiques augmente leur potentiel invasif et leur capacité à envahir les vaisseaux sanguins dans un modèle murin [10]. Plusieurs études in vitro ont démontré que l'OPN stimule la migration des cellules de nombreux types de cancers, dont le mélanome [15], les cancers du sein [16] et de la prostate [17]. Les trois isoformes $a, b$ et $c$ de l'OPN sont surexprimées au niveau de certains cancers, comme les gliomes de haut grade, avec une prédominance de la forme $b$ qui augmente le potentiel invasif des cellules [18]. De la même façon, la transfection d'une lignée cellulaire cancéreuse mammaire, afin d'y surexprimer la BSP, stimule la migration et l'invasion in vitro [19].

De nombreuses études décrivent les mécanismes par lesquels les protéines de la famille des SIBLING participent à la dégradation de la matrice extracellulaire. L'intervention d'enzymes spécifiques régule les propriétés pro-invasives et pro-migratoires des protéines SIBLING. Par exemple, l'invasion des cellules de carcinomes hépatocellulaires est induite par des peptides de l'OPN clivée par la MMP9 (métalloprotéinase matricielle 9) et la thrombine [20]. Par ailleurs, la transglutamination de I'OPN entraînerait la formation de polymères inactifs et résistants à la protéolyse qui inhibent l'invasion et la migration des cellules cancéreuses mammaires in vitro [21]. En plus d'être clivées par les MMP, les SIBLING jouent un rôle dans l'activation de ces enzymes. La BSP, la DMP1 et l'OPN se lient et régulent l'activité des MMP2, MMP9 et MMP3, respectivement [22]. De façon remarquable, l'expression des SIBLING est corrélée à celle de leurs MMP respectives au niveau de nombreux types de cancers $[23,24]$. Les SIBLING activent les MMP en transformant les proMMP en forme enzymatique active ou en réactivant des MMP maintenues sous leur forme inactive par les inhibiteurs tissulaires des MMP (TIMP) [22]. La BSP favorise l'invasion de lignées cellulaires cancéreuses à tropisme osseux in vitro en adressant la MMP2 à la surface cellulaire via les intégrines $\alpha v \beta 3$ [25]. La DMP1 


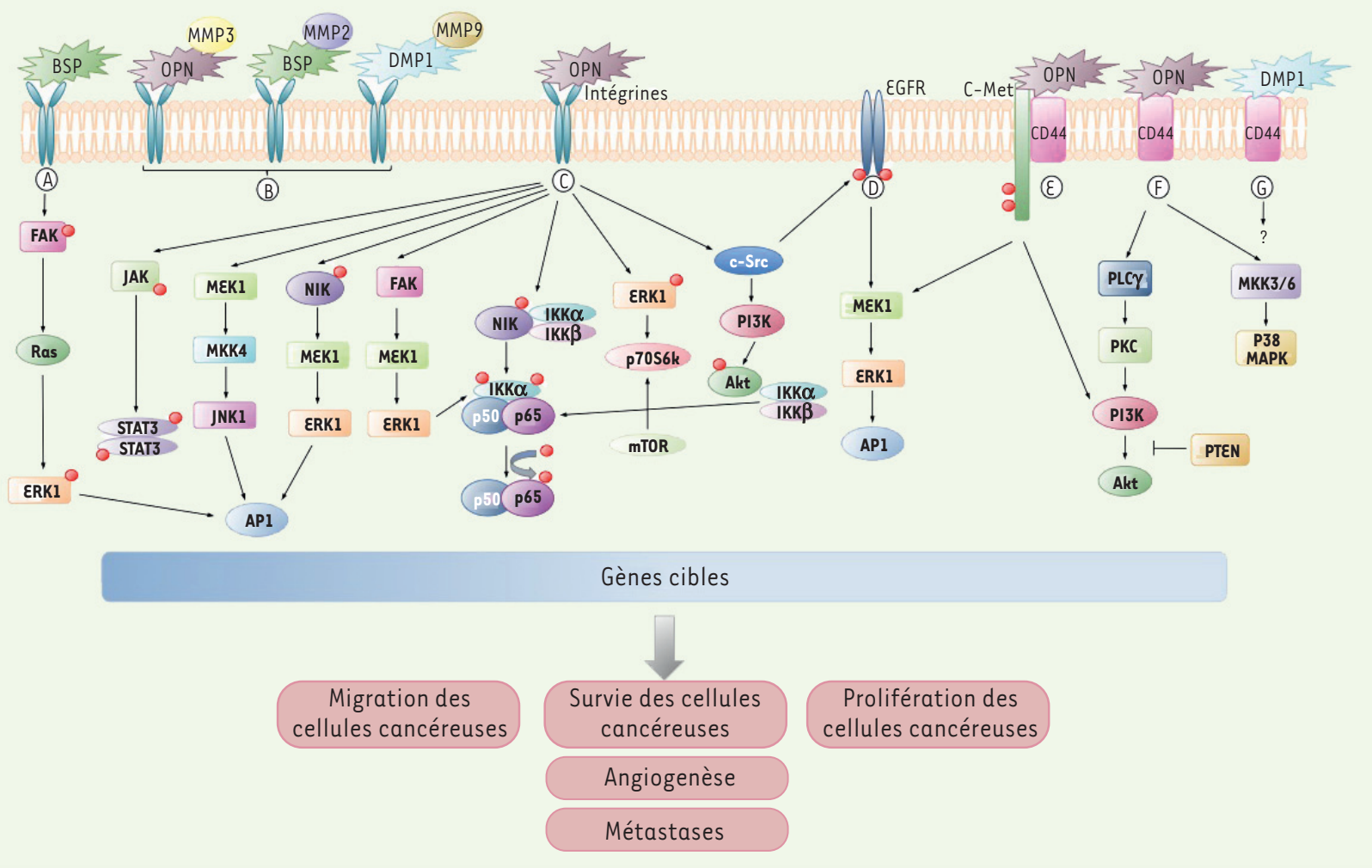

Figure 3. Voies de signalisation activées par les SIBLING au niveau des cellules cancéreuses. La plupart des protéines SIBLING peuvent interagir avec les récepteurs de surface cellulaire de type intégrine grâce à leur domaine RGD (voies A, B et C). L'OPN et la DMPl ont été décrites également comme pouvant interagir avec le récepteur CD44 (voies $\varepsilon$, F et G). La liaison à ces récepteurs conduit à l'activation de voies de signalisation aboutissant à la régulation de gènes impliqués dans le contrôle de la croissance tumorale, la migration cellulaire, l'angiogenèse et la formation de métastases. La liaison de l'OPN au CD44 stimule la migration et l'invasion cellulaire via la cascade de kinases (voie F). L'OPN, via sa liaison à CD44, peut également activer c-Met, induisant la phosphorylation et l'activation de ERK (extracellular signal-regulated kinases) et Akt (voie $\varepsilon$ ). L’OPN, en se liant à l'intégrine $\alpha v \beta 3$ stimule la migration et l'invasion des cellules cancéreuses via l'activation de voies de signalisation aboutissant à une activation de la croissance tumorale (voie C) (pour une revue détaillée des voies de signalisation activées par l'OPN, voir [12]). La liaison de la BSP à l'intégrine $\alpha v \beta 3$ entraîne une invasion accrue des cellules tumorales via l'activation de FAK (focal adhesion kinase) et la phophorylation de ERK (extracellular signal-regulated kinase) (voie A). Les voies de signalisation affectées par les autres membres de la famille des SIBLING au niveau des cellules cancéreuses ne sont pas encore connues (voie G). Enfin, l'OPN, la BSP et la DMPl peuvent favoriser la migration et l'invasion des cellules cancéreuses via la liaison et l'activation des MMP (voie B). Les phosphorylations sont représentées par des points rouges. STAT3: signal transducer and activator of transcription 3 ; JAK : Janus kinase ; JNK1 : c-jun N-terminal kinase; API : activating protein 1 ; NIK : nuclear factor $K B$-inducing kinase; IKK : IKB kinase ; mTOR : mammalian target of rapamycin; PLC $\gamma$ : phospholipase $C \gamma$; PKC : protéine kinase $C$; PTEN : phosphatase and tensin homolog.

lie la MMP9 aux intégrines, et peut-être au CD44, et augmente ainsi le potentiel invasif des cellules de cancer du côlon in vitro [26].

\section{Échappement au système du complément}

Les SIBLING apparaissent comme des régulateurs essentiels du potentiel métastatique des cellules cancéreuses. Au cours du long et périlleux parcours de la cascade métastatique (Figure 2), la survie des cellules tumorales requiert de contourner de façon efficace le système immunitaire de l'hôte. Un acteur important du système immunitaire inné est le système du complément. II a été démontré que I'OPN, la DMPl et la BSP peuvent se lier à un élément du système du complément, le facteur $\mathrm{H}$, pour former des complexes de haut poids molécu- laire détectables dans le sérum [27, 28]. Cette liaison au facteur $\mathrm{H}$ s'oppose à la formation du complexe d'attaque membranaire et protège ainsi les cellules cancéreuses qui expriment les SIBLING de la lyse induite par le système du complément (Figure 2, étape 5).

\section{Formation de métastases}

La formation de métastases est un processus complexe qui conduit à l'implantation et à la prolifération de cellules cancéreuses à des sites secondaires distants. Ce processus implique l'évasion des cellules tumorales de la tumeur primaire, leur passage dans des capillaires 


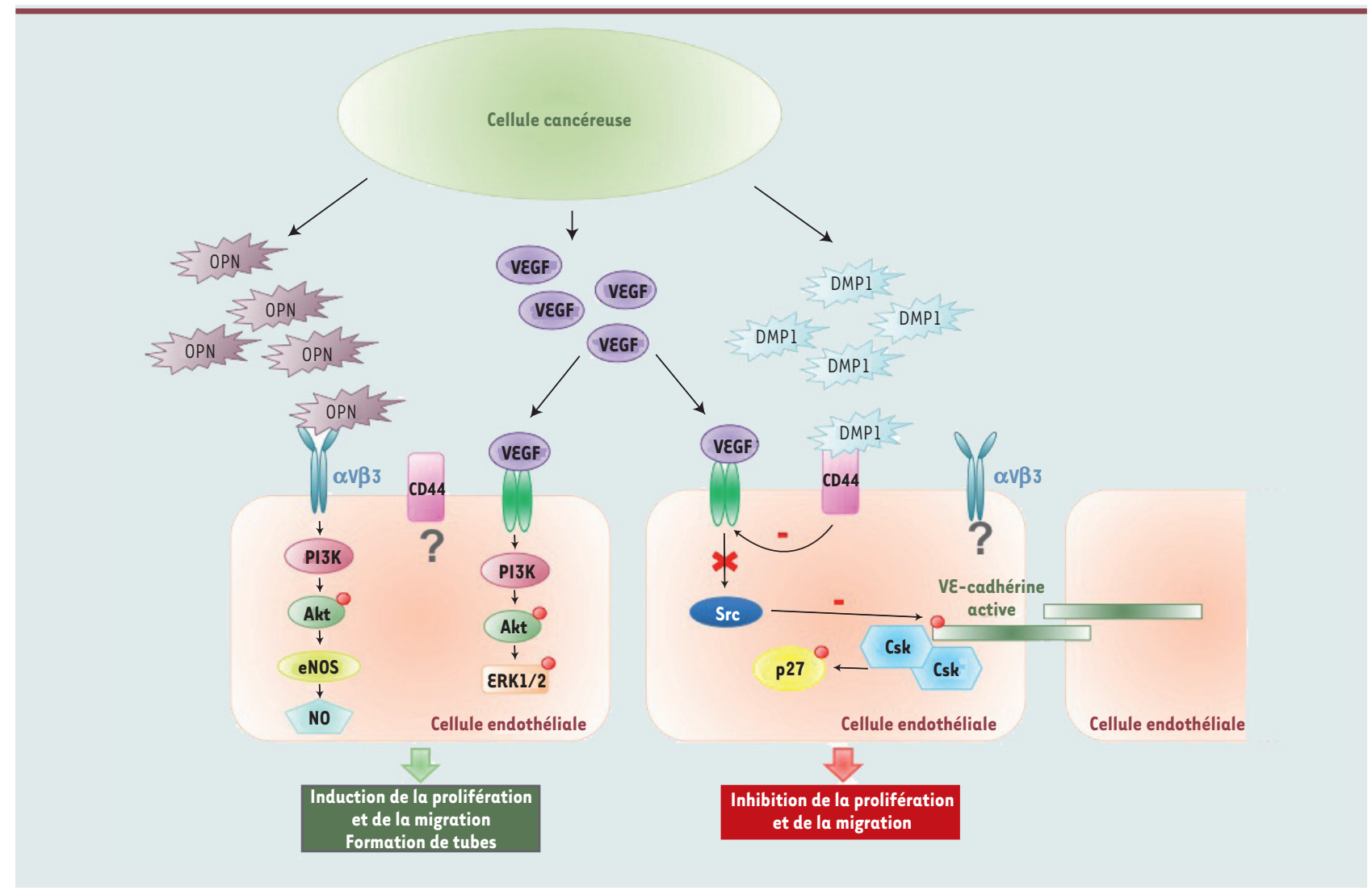

Figure 4. Rôle des SIBLING dans l'angiogenèse. Les cellules cancéreuses sécrètent du VEGF (vascular endothelial growth factor) qui se lie à son récepteur principal, le VEGFR-2, à la surface des cellules endothéliales, induisant ainsi l'activation de la voie PI3K/Akt/ERK1/2 (extracellular signal-regulated kinases 1/2) dans ces cellules, ainsi que la prolifération et la migration de ces cellules. L'OPN et la DMPl sont capables de renforcer ou d'inhiber la signalisation du VEGF-VEGFR-2, respectivement. L'OPN, en se liant au récepteur $\alpha v \beta 3$, active la voie PI3K/Akt menant à la production de monoxyde d'azote (NO) via l'activation de l'eNOS (endothelial NO synthase). Le NO va stimuler la prolifération et la migration des cellules endothéliales, ainsi que la formation de tubes. Contrairement à l'OPN, la DMPI agit comme une molécule anti-angiogénique. La liaison du VEGF au VEGF-R2 active la kinase Src qui inactive la VE-cadhérine (vascular endothelial-cadherin), un élément essentiel des jonctions adhérentes entre les cellules endothéliales. S'opposant à l'action du VEGF, la DMPI qui se lie au récepteur CD44, induit une augmentation de l'expression de la VE-cadhérine membranaire. La VEcadhérine active la kinase Csk (C-Src kinase) et induit p27, un inhibiteur du cycle cellulaire, et donc mime le processus d'inhibition de croissance par contact. La VE-cadhérine est aussi capable d'inhiber la phosphorylation du VEGFR-2 et ainsi d'inactiver toute la voie de signalisation en aval. De cette façon, la DMPlinterfère avec la voie du VEGF pour inhiber la prolifération et la migration des cellules endothéliales.

sanguins et/ou lymphatiques, et leur prolifération au niveau d'un site distant pour générer une tumeur secondaire ou métastase (pour revue, voir [29]). Les SIBLING pourraient jouer un rôle capital dans la formation des métastases car elles sont impliquées, comme décrit précédemment, dans l'adhésion cellulaire, la migration et la dégradation de la matrice.

II a été démontré dans plusieurs études que l'OPN et la BSP jouent un rôle prométastatique (pour revue, voir [30]). Les tumeurs susceptibles d'évoluer vers un stade plus avancé présentent une expression de SIBLING augmentée ou apparaissant de novo. La surexpression de I'OPN dans des cellules épithéliales mammaires de rat Rama 37 non métastatiques induit le développement de métastases pulmonaires chez $55 \%$ des animaux chez lesquels la xénogreffe sous-cutanée des cellules a entraîné le développement d'une tumeur primaire [31]. À l'inverse, son inhibition à l'aide d'oligonucléotides anti-sens dans les cellules cancéreuses mammaires MDA-MB-231 réduit la formation de métastases osseuses [32]. De plus, il a été démontré récemment que l'OPN exogène joue un rôle important dans la formation de métastases à partir de cellules de mélanome et d'ostéosarcome humains [33]. L'expression élevée de SIBLING est associée aux cancers ostéotropiques, comme par exemple le cancer du sein [34] ou de la prostate [35]. Une étude de génomique fonctionnelle a décrit dans les cellules de cancer du sein une signature favorable au développement de métastases osseuses comprenant l'OPN et l'interleukine 11 associées, soit au récepteur de la chimiokine CXCR4, soit au facteur de croissance des tissus conjonctifs (CTGF) dans les cellules de cancer du sein [36]. Aussi, la surexpression de la BSP dans les cellules MDA-MB-231 
augmente la formation de métastases osseuses [37]. L'affinité pour I'os des cellules cancéreuses qui expriment la BSP a été validée dans une étude où la seule surexpression de la BSP dans des cellules cancéreuses mammaires qui métastasent au cerveau est suffisante pour induire leur tropisme osseux, alors qu'aucune lésion osseuse n'est observée avec les cellules contrôles [38]. L'expression des SIBLING par les carcinomes du sein et de la prostate a permis de proposer I'hypothèse selon laquelle les cellules cancéreuses peuvent acquérir un phénotype ostéoblastique et dont l'adhésion, la prolifération et/ou la survie sont favorisées au niveau du tissu osseux $[39,40]$. II est remarquable que des facteurs de transcription essentiels à l'expression des gènes impliqués dans l'identité osseuse, tels que Runx2 et osterix, sont aussi exprimés au niveau des cellules cancéreuses mammaires et y régulent l'expression de certaines SIBLING [41].

\section{Angiogenèse}

La tumeur est dépendante d'un apport constant en oxygène et en nutriments via la circulation sanguine pour sa croissance et pour sa dissémination potentielle. Le récepteur de type intégrine $\alpha v \beta 3$ est surexprimé à la surface des cellules endothéliales activées et joue un rôle clé dans le processus d'angiogenèse [42]. Différentes études ont démontré que les SIBLING, et notamment I'OPN et la BSP, sont des molécules pro-angiogéniques. L'OPN interagit via son domaine RGD avec les intégrines $\alpha v \beta 3$ présentes à la surface des cellules endothéliales et induit la voie de signalisation PI3K (phosphoinositide 3-kinase)/Akt (protéine kinase B) menant à l'activation de l'eNOS (endothelial NO synthase) et à la production de NO (monoxyde d'azote). Celui-ci induit la prolifération et la migration des cellules endothéliales ainsi que la formation de tubes [43]. L'influence de I'OPN sur la genèse de nouveaux capillaires infiltrant les tumeurs a été démontrée dans de nombreux modèles in vitro et in vivo [44, 45]. Une étude récente suggère que seule l'OPNa serait capable d'induire la sécrétion de VEGF (vascular endothelial growth factor) au niveau des cellules cancéreuses pulmonaires. L'OPNc aurait plutôt tendance à diminuer la production de VEGF, et I'OPNb n'aurait pas d'effet sur cette sécrétion [46]. Des expériences ont démontré que le blocage de la liaison des SIBLING aux intégrines est une approche prometteuse pour l'inhibition de l'angiogenèse et la croissance tumorale associée. Par exemple, le blocage de la liaison de l'OPNa inhibe l'angiogenèse et empêche le développement de cancer pulmonaire chez la souris [44]. Sur la base de la présence d'un motif RGD au niveau de leur séquence primaire, les autres protéines SIBLING sont également susceptibles d'interagir avec les cellules endothéliales. La BSP favorise la formation de nouveaux vaisseaux dans un modèle de membrane chorioallantoïdienne (CAM) et ce, via les intégrines $\alpha v \beta 3$ [47].

Contrairement à I'OPN et à la BSP, nous avons récemment montré que la protéine DMPl possède des propriétés anti-angiogéniques. En effet, la DMPl est capable d'interférer avec l'activation des cellules HUVEC (human umbilical vein endothelial cells) par le VEGF en empêchant la phosphorylation de son principal récepteur, le VEGFR-2 [48]. Ainsi deux protéines de la famille SIBLING, I'OPN et la DMPl, en se liant à leurs récepteurs de surface cellulaire au niveau des cellules endothéliales, activent des voies de signalisation différentes et induisent des effets complètement opposés sur le processus complexe qu'est l'angiogenèse (Figure 4). Cette observation n'est probablement pas sans rapport avec le fait que l'expression de l'OPN par les cellules cancéreuses est largement corrélée à un mauvais pronostic, alors que l'expression de DMPl au niveau de la tumeur primaire est plutôt un facteur de bon augure pour les patientes présentant un cancer du sein [49].

\section{Conclusion}

Depuis la première mise en évidence de la surexpression ectopique des protéines SIBLING au niveau de différents types de cancers humains, nos connaissances ont beaucoup évolué quant à la régulation de leur expression et à leur mode d'action grâce à l'effort conjoint des chercheurs dans ce domaine. L'intérêt actuel de ces derniers se concentre sur la détection sérique de ces protéines et leur utilisation comme marqueurs diagnostiques et pronostiques potentiels du cancer. Ainsi, un niveau sérique élevé d'OPN est un marqueur avéré de progression métastatique chez les patients atteints de cancers du sein, de la prostate ou du rein. Les concentrations de BSP et de DSPP sont augmentées dans le sérum des patients cancéreux. En particulier, le taux de DSPP circulante est significativement différent chez un sujet sain et un sujet présentant une tumeur de bas grade. Au vu de leurs fonctions diverses en relation avec la progression cancéreuse et métastatique, l'autre attrait des protéines SIBLING réside dans le fait qu'elles représentent des cibles prometteuses pour la thérapie anticancéreuse. À ce jour, plusieurs études utilisant des modèles animaux ont démontré que l'inhibition de l'expression des SIBLING, ou l'interférence avec leurs récepteurs spécifiques, permet d'inhiber significativement la progression tumorale et métastatique. $\diamond$

\section{SUMMARY}

SIBLING proteins: molecular tools for tumor progression and angiogenesis

The small integrin-binding ligand $\mathrm{N}$-linked glycoprotein (SIBLING) family consists of osteopontin (OPN), bonesialoprotein (BSP), dentin matrix protein 1 (DMP1), dentin sialophosphoprotein (DSPP) and matrix extracellular phosphoglycoprotein (MEPE). These proteins, initially identified in bone and teeth, share many structural characteristics. It is now well established that they are over expressed in many tumors and play a critical role at different steps of cancer development. In this review, we 
describe the roles of SIBLING proteins at different stages of cancer progression including cancer cell adhesion, proliferation, migration, invasion, metastasis and angiogenesis. $\diamond$

\section{LIENS D'INTÉRÊT}

Les auteurs déclarent n'avoir aucun lien d'intérêt concernant les données publiées dans cet article.

\section{RÉFÉRENCES}

1. Fisher LW, Fedarko NS. Six genes expressed in bones and teeth encode the current members of the SIBLING family of proteins. Connect Tissue Res $2003 ; 44: 33-40$.

2. Bellahcene A, Castronovo V, Ogbureke KU, et al. Small integrin-binding ligand $\mathrm{N}$-linked glycoproteins (SIBLINGs) : multifunctional proteins in cancer. Nat Rev Cancer 2008 ; 8 : 212-26.

3. Staines KA, MacRae VE, Farquharson C. The importance of the SIBLING family of proteins on skeletal mineralisation and bone remodelling. J Endocrinol $2012 ; 214: 241-55$.

4. El-Tanani MK, Campbell FC, Kurisetty V, et al. The regulation and role of osteopontin in malignant transformation and cancer. Cytokine Growth Factor Rev 2006 ; 17 : 463-74.

5. Hanahan D, Weinberg RA. Hallmarks of cancer : the next generation. Cell $2011 ; 144: 646-74$.

6. Sung V, Stubbs JT, 3rd, Fisher L, et al. Bone sialoprotein supports breast cancer cell adhesion proliferation and migration through differential usage of the alpha $(\mathrm{v})$ beta 3 and alpha $(\mathrm{v})$ beta 5 integrins. J Cell Physiol $1998 ; 176$ : 482-94.

7. Lee JL, Wang MJ, Sudhir PR, et al. Osteopontin promotes integrin activation through outside-in and inside-out mechanisms : OPN-CD44V interaction enhances survival in gastrointestinal cancer cells. Cancer Res $2007 ; 67: 2089-97$.

8. Pecheur I, Peyruchaud 0 , Serre CM, et al. Integrin alpha(v)beta3 expression confers on tumor cells a greater propensity to metastasize to bone. Faseb J $2002 ; 16: 1266-8$.

9. von Marschall Z, Fisher LW. Dentin matrix protein-1 isoforms promote differential cell attachment and migration. J Biol Chem 2008 ; $283: 32730-40$.

10. Khodavirdi AC, Song Z, Yang S, et al. Increased expression of osteopontin contributes to the progression of prostate cancer. Cancer Res $2006 ; 66: 883-8$.

11. Courter D, Cao H, Kwok S, et al. The RGD domain of human osteopontin promotes tumor growth and metastasis through activation of survival pathways. PLoS One $2010 ; 5:$ e9633.

12. Ahmed M, Behera R, Chakraborty G, et al. Osteopontin : a potentially important therapeutic target in cancer. Expert Opin Ther Targets 2011 ; 15 : 1113-26.

13. Kayed H, Kleeff J, Keleg S, et al. Effects of bone sialoprotein on pancreatic cancer cell growth, invasion and metastasis. Cancer Lett 2007 ; 245 : 171-83.

14. Joshi R, Tawfik A, Edeh N, et al. Dentin sialophosphoprotein (DSPP) gene-silencing inhibits key tumorigenic activities in human oral cancer cell line, OSC2. PLoS One $2010 ; 5$ : el3974.

15. Rangaswami H, Kundu GC. Osteopontin stimulates melanoma growth and lung metastasis through NIK/MEKK1-dependent MMP-9 activation pathways. Oncol Rep 2007 ; 18 : 909-15.

16. Khan $S A$, Cook AC, Kappil M, et al. Enhanced cell surface CD44 variant (v6, v9) expression by osteopontin in breast cancer epithelial cells facilitates tumor cell migration : novel posttranscriptional, post-translational regulation. Clin Exp Metastasis $2005 ; 22$ : 663-73.

17. Irby RB, McCarthy SM, Yeatman TJ. Osteopontin regulates multiple functions contributing to human colon cancer development and progression. Clin Exp Metastasis $2004 ; 21$ : 515-23.

18. Yan W, Pian C, Zhao P, et al. Expression pattern of osteopontin splice variants and its functions on cell apoptosis and invasion in glioma cells. Neuro Oncol $2010 ; 12: 765-75$.

19. Sharp JA, Waltham M, Williams ED, et al. Transfection of MDA-MB-231 human breast carcinoma cells with bone sialoprotein (BSP) stimulates migration and invasion in vitro and growth of primary and secondary tumors in nude mice. Clin Exp Metastasis 2004 ; 21 : 19-29.

20. Takafuji V, Forgues $M$, Unsworth $\varepsilon$, et al. An osteopontin fragment is essential for tumor cell invasion in hepatocellular carcinoma. Oncogene $2007 ; 26: 6361-71$.

21. Mangala LS, Arun B, Sahin AA, Mehta K. Tissue transglutaminase-induced alterations in extracellular matrix inhibit tumor invasion. Mol Cancer $2005 ; 4: 33$.

22. Fedarko NS, Jain A, Karadag A, Fisher LW. Three small integrin binding ligand N-linked glycoproteins (SIBLINGS) bind and activate specific matrix metalloproteinases. Faseb J 2004 ; 18 : 734-6.

23. Fisher LW, Jain A, Tayback M, Fedarko NS. Small integrin binding ligand N-linked glycoprotein gene family expression in different cancers. Clin Cancer Res 2004 ; 10: 8501-11.

24. Ogbureke KU, Nikitakis NG, Warburton G, et al. Up-regulation of SIBLING proteins and correlation with cognate MMP expression in oral cancer. Oral Oncol $2007 ; 43: 920-32$.

25. Karadag A, Ogbureke KU, Fedarko NS, Fisher LW. Bone sialoprotein, matrix metalloproteinase 2, and alpha(v)beta3 integrin in osteotropic cancer cell invasion. J Natl Cancer Inst 2004 ; 96 : 956-65.

26. Karadag A, Fedarko NS, Fisher LW. Dentin matrix protein 1 enhances invasion potential of colon cancer cells by bridging matrix metalloproteinase- 9 to integrins and CD44. Cancer Res $2005 ; 65$ : 11545-52.

27. Fedarko NS, Fohr B, Robey PG, et al. Factor $H$ binding to bone sialoprotein and osteopontin enables tumor cell evasion of complement-mediated attack. J Biol Chem $2000 ; 275$ : 16666-72.
28. Jain A, Karadag A, Fohr B, et al. Three SIBLINGs (small integrin-binding ligand, $\mathrm{N}$-linked glycoproteins) enhance factor H's cofactor activity enabling MCP-like cellular evasion of complement-mediated attack. J Biol Chem $2002 ; 277: 13700-8$

29. Fidler IJ. The pathogenesis of cancer metastasis : the 'seed and soil' hypothesis revisited. Nat Rev Cancer $2003 ; 3: 453-8$.

30. Wai Py, Kuo PC. Osteopontin : regulation in tumor metastasis. Cancer Metastasis Rev $2008 ; 27: 103-18$.

31. Oates AJ, Barraclough R, Rudland PS. The identification of osteopontin as a metastasis-related gene product in a rodent mammary tumour model. Oncogene 1996 ; 13 : 97-104.

32. Adwan H, Bauerle TJ, Berger MR. Downregulation of osteopontin and bone sialoprotein II is related to reduced colony formation and metastasis formation of MDA-MB-231 human breast cancer cells. Cancer Gene Ther $2004 ; 11: 109-20$.

33. Mandelin J, Lin EC, Hu DD, et al. Extracellular and intracellular mechanisms that mediate the metastatic activity of exogenous osteopontin. Cancer $2009 ; 115: 1753-64$.

34. Bellahcene A, Merville MP, Castronovo V. Expression of bone sialoprotein, a bone matrix protein, in human breast cancer. Cancer Res 1994 ; 54 : 2823-6.

35. Waltregny D, Bellahcene A, Van Riet I, et al. Prognostic value of bone sialoprotein expression in clinically localized human prostate cancer. J Natl Cancer Inst $1998 ; 90: 1000-8$.

36. Kang $Y$, Siegel PM, Shu W, et al. A multigenic program mediating breast cancer metastasis to bone. Cancer Cell $2003 ; 3: 537-49$.

37. Zhang JH, Tang J, Wang J, et al. Over-expression of bone sialoprotein enhances bone metastasis of human breast cancer cells in a mouse model. Int J Oncol $2003 ; 23: 1043-8$.

38. Zhang JH, Wang J, Tang J, et al. Bone sialoprotein promotes bone metastasis of a non-bone-seeking clone of human breast cancer cells. Anticancer Res $2004 ; 24: 1361-8$.

39. Koeneman KS, Yeung F, Chung LW. Osteomimetic properties of prostate cancer cells : a hypothesis supporting the predilection of prostate cancer metastasis and growth in the bone environment. Prostate 1999; 39: 246-61.

40. Bellahcene A, Bachelier R, Detry C, et al. Transcriptome analysis reveals an osteoblast-like phenotype for human osteotropic breast cancer cells. Breast Cancer Res Treat $2007 ; 101: 135-48$.

41. Barnes GL, Javed A, Waller SM, et al. Osteoblast-related transcription factors Runx2 (Cbfal/AML3) and MSX2 mediate the expression of bone sialoprotein in human metastatic breast cancer cells. Cancer Res $2003 ; 63: 2631-7$.

42. Brooks PC, Clark RA, Cheresh DA. Requirement of vascular integrin alpha $v$ beta 3 for angiogenesis. Science 1994 ; $264: 569-71$.

43. Wang $Y$, Yan W, Lu X, et al. Overexpression of osteopontin induces angiogenesis of endothelial progenitor cells via the avbeta3/PI3K/AKT/ eNOS/NO signaling pathway in glioma cells. Eur J Cell Biol $2011 ; 90: 642-8$.

44. Cui $R$, Takahashi F, Ohashi $R$, et al. Abrogation of the interaction between osteopontin and alphavbeta 3 integrin reduces tumor growth of human lung cancer cells in mice. Lung Cancer 2007 ; 57 : 302-10.

45. Dai J, Peng L, Fan K, et al. Osteopontin induces angiogenesis through activation of PI3K/AKT and ERKl/2 in endothelial cells. Oncogene $2009 ; 28$ : 3412-22.

46. Blasberg JD, Goparaju CM, Pass HI, Donington JS. Lung cancer osteopontin isoforms exhibit angiogenic functional heterogeneity. J Thorac Cardiovasc Surg $2010 ; 139: 1587-93$.

47. Bellahcene A, Bonjean K, Fohr B, et al. Bone sialoprotein mediates human endothelial cell attachment and migration and promotes angiogenesis. Circ Res $2000 ; 86: 885-91$.

48. Pirotte $S$, Lamour V, Lambert V, et al. Dentin matrix protein 1 induces membrane expression of $V \mathcal{E}$-cadherin on endothelial cells and inhibits VEGFinduced angiogenesis by blocking VEGFR-2 phosphorylation. Blood 2011 ; $117: 2515-26$.

49. Bucciarelli $\varepsilon$, Sidoni A, Bellezza G, et al. Low dentin matrix protein 1 expression correlates with skeletal metastases development in breast cancer patients and enhances cell migratory capacity in vitro. Breast Cancer Res Treat $2007 ; 105: 95-104$

50. Chabas D. L'ostéopontine, une molécule aux multiples facettes. Med Sci (Paris) $2005 ; 21: 832-8$.

51. Orian-Rousseau V. CD44, un régulateur de la croissance et de l'invasion cellulaire. Med Sci (Paris) 2003 ; $19: 405-7$.

52. Bidard FC, Poupon MF. Biologie du processus métastatique. Med Sci (Paris) $2012 ; 28: 89-95$

\section{TIRÉS À PART}

A. Bellahcène 